\title{
Improvements in Performance of Broadband Helix Traveling-Wave Tubes
}

\author{
Tushar K. Ghosh, Member, IEEE, Anthony J. Challis, Alan Jacob, Darrin Bowler, \\ and Richard G. Carter, Senior Member, IEEE
}

\begin{abstract}
The effectiveness of positive phase velocity tapering to improve the performance of a broadband helix traveling-wave tube (TWT) has been investigated. A large-signal model of an existing 4.5-18 GHz two-section mini-TWT with uniform pitch has been validated against the experimental results, and used as a starting point to design and develop a number of TWTs with different taper lengths. Comparisons between the experimental results of uniform pitch TWT and positively tapered TWTs show significant improvements in output power, electronic efficiency, and second harmonic content throughout the operating frequency band for the positively tapered TWTs. Increases in electronic efficiency and output power up to $5.2 \%$ points and $50 \mathrm{~W}(1.7 \mathrm{~dB})$, respectively, have been achieved.
\end{abstract}

Index Terms-Broadband amplifiers, efficiency, harmonic, helix, phase velocity tapering, traveling-wave tube (TWT).

\section{INTRODUCTION}

$\mathbf{0}$ VER THE last three decades, the broadband helix traveling-wave tube (TWT) has gained ascendancy in radar, electronic countermeasures, and other warfare systems [1]. Increases in output power and bandwidth of the device are essential to meet the ever-increasing demands for these applications.

High dispersion of the slow-wave structure (SWS) is one of the limiting factors in achieving high efficiency over a multioctave band [2]-[4]. Other criticalities involve low interaction impedance and high RF loss at the higher end of the operating frequency band [5]-[8]. Considerable efforts have been made in the past to overcome these limitations. A major area of research was to investigate the effects of different shapes of metal vanes and dielectric support rods in controlling the dispersion [9]-[11]. In other attempts, the optimization of vane radius to achieve a potentially dispersion-free characteristic while maintaining the interaction impedance at the higher end of the frequency band was carried out [12], [13]. Studies on semivane structure and dielectric constant of the material used for the support rods have shown their effectiveness in shaping the phase velocity to obtain a nearly flat dispersion [14], [15]. However, it is the phase lag of the RF current wave with respect to the circuit wave toward the end of the SWS that restricts the amount of energy being trans-

Manuscript received August 14, 2007; revised October 25, 2007. The review of this paper was arranged by Editor W. Menninger.

T. K. Ghosh, A. J. Challis, A. Jacob, and D. Bowler are with the e2v Technologies, Chelmsford CM1 2QU, U.K. (e-mail: tushar.ghosh@e2v.com; tony.challis@e2v.com; alan.jacob@e2v.com; darrin.bowler@e2v.com).

R. G. Carter is with the Department of Engineering, Lancaster University, Lancaster LA1 4YR, U.K. (e-mail: r.carter@lancaster.ac.uk).

Color versions of one or more of the figures in this paper are available online at http://ieeexplore.ieee.org.

Digital Object Identifier 10.1109/TED.2007.913006

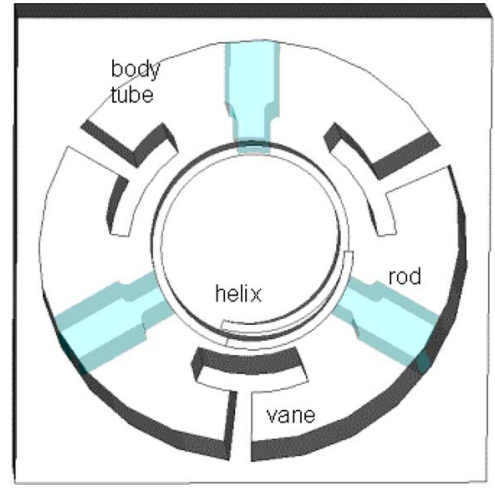

Fig. 1. Microwave studio model of the single-turn helix with T-shaped metallic vanes and dielectric support rods.

ferred from the beam to the RF wave. The loss of synchronism between the wave and the beam limits the output power, and hence, the efficiency of the TWTs. In a narrow-band TWT, a helix pitch profile, which incorporates a section with a positive phase velocity followed by a negative phase velocity taper, can be used at the output section of the SWS to resynchronize the wave with the beam, and thereby, achieve high efficiency [16]. An alternative pitch profile, a positively tapered helix, which was first conceived in the 1960s, can also be used for this purpose [17]. In a recent work, a theoretical study on its usefulness in improving the wideband performance was carried out [18]. The experimental performance of a multioctave band positively tapered helix mini-TWT was first demonstrated by Ghosh et al. [19]. In this paper, the design strategy of such devices is elaborated upon and supported by further experimental results. An extensive literature survey is also included in the introduction.

\section{LARGe-Signal Model VALIDATION}

To design and accurately predict the performance of a positively tapered TWT, it is useful to have a validated model of a baseline TWT such as the one with uniform pitch. For this purpose, an existing two-section $4.5-18 \mathrm{GHz}$ helix mini-TWT, designated TWT1, with uniform pitch was used as the starting point of the design. The tube was fitted with a two-stage collector and subminiature A (SMA) connectors on both the input and output ports. The operating voltage and beam current of TWT1 are $4.5 \mathrm{kV}$ and $200 \mathrm{~mA}$, respectively.

A large-signal interaction code [21] was initially used to validate the model of TWT1 and later used to design positive tapers. To generate a set of values for Pierce impedance and propagation constant at different frequencies of the operating band, which were taken as input to the code, a model of the SWS similar to Fig. 1 was simulated using the 3-D electromagnetic field 


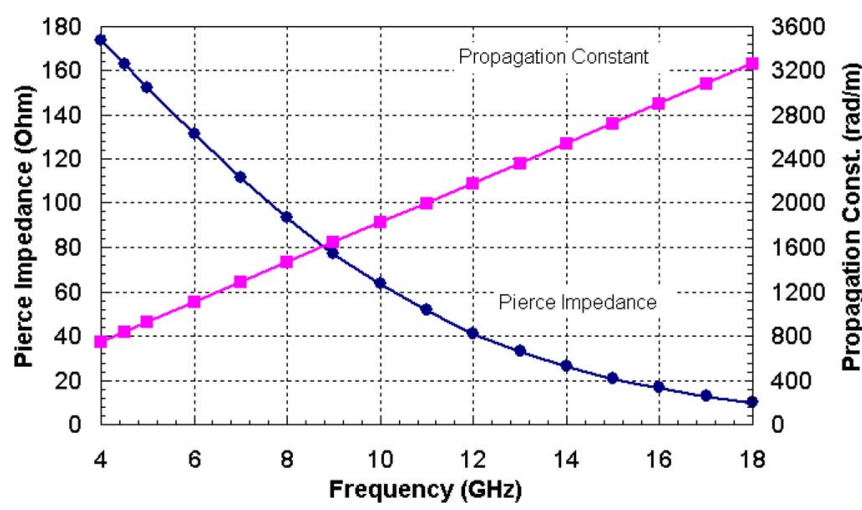

Fig. 2. Pierce impedance and propagation constant of TWT1.

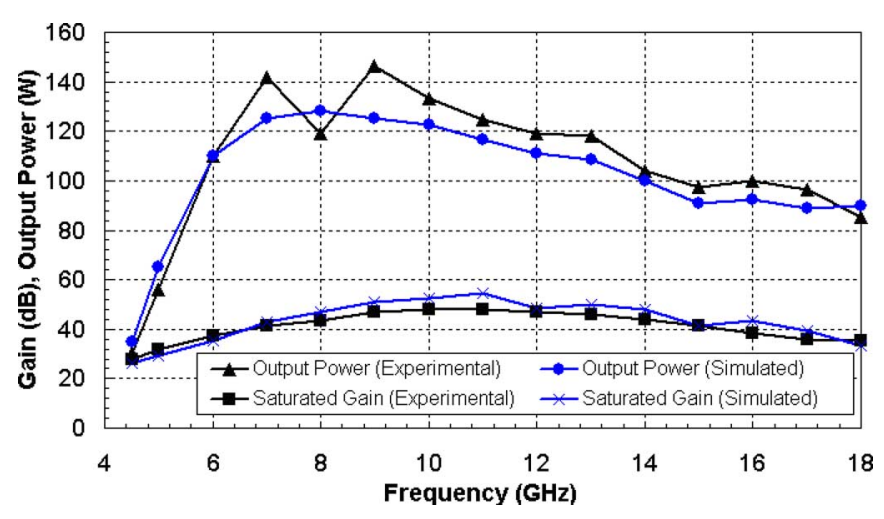

Fig. 3. Comparison between simulated and experimental output of TWT1.

simulator Microwave Studio. ${ }^{1}$ This model consists of a singleturn helix with finite tape thickness, dielectric support rods, T-shaped metallic vanes, and body tube. The dielectric constant of the materials were defined to ensure accurate computation of the impedance and phase velocity of the wave. Periodic boundary conditions were applied at both ends of the structure along the axial direction of the helix. Once the steady state was reached in the eigenmode solver, the values of Pierce impedance and propagation constant at different frequencies were calculated using the postprocessor, as shown in Fig. 2. As expected, the Pierce impedance decreases and propagation constant increases with the increase in frequency. Other than these two parameters, the dimensions of the SWS, basic electrical parameters (beam voltage, beam current, and beam radius), return loss, and cold loss (attenuator, sever, and helix) were used as input to perform the large-signal analysis of TWT1. To achieve the beam parameters, the electron gun and the periodic permanent magnet (PPM) focusing system were modeled assuming uniform current density in the beam and validated against the experimental results. The good agreement between the simulated and the experimental values of saturated output power and saturated gain in Fig. 3 validates the large-signal model. The slight mismatch in the output power may be attributed to the approximation of

${ }^{1}$ Supplied by Computer Simulation Technologies (CST).

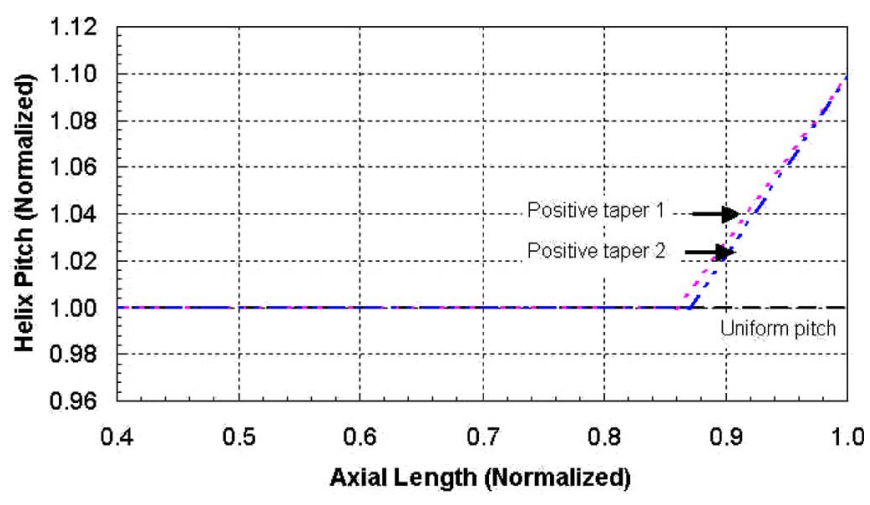

Fig. 4. Different pitch profiles.

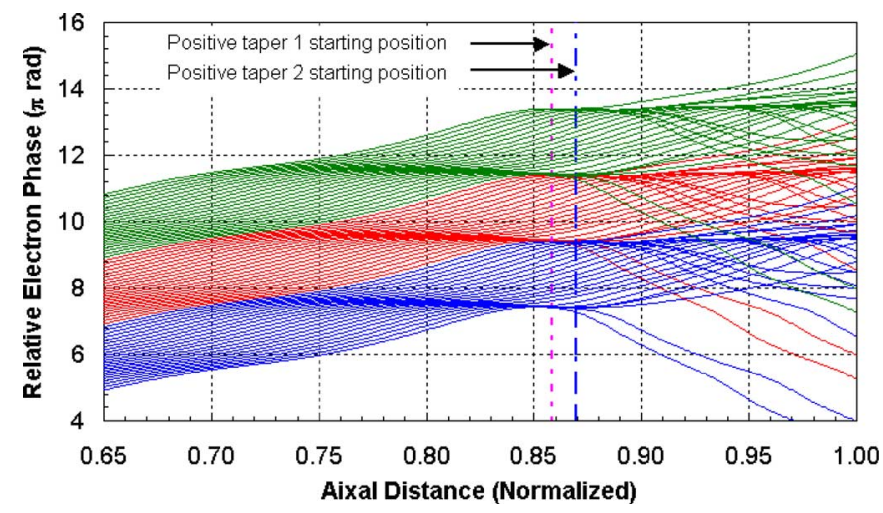

Fig. 5. Applegate diagram of TWT1 at $18 \mathrm{GHz}$ showing the positive taper starting positions.

uniform charge density in the beam and to the assumption of constant return loss of the output coupler at all frequencies. Another parameter that may have contributed to the mismatch is a slightly higher helix cold loss [7].

\section{POSITIVE TAPER DESIGN}

To optimize the performance, a number of TWTs were designed and developed to have different taper dimensions [20], three of which are discussed here. In a positively tapered helix, the pitch remains constant over a length and gradually increases toward the end, as shown in Fig. 4. The length of the taper is a small fraction of the total length of the helix between the input and output ports, and is determined from the Applegate diagram, as shown in Fig. 5. The validated model of TWT1 in the largesignal interaction code was used to generate the relative phase of the electrons against the normalized axial length of the helix at $18 \mathrm{GHz}$. It was intended to use the calculations at $18 \mathrm{GHz}$ as the basis for the design, because the output power in TWT1 decreases at the higher end of the operating frequency band (see Fig. 3). It is evident from the plot in Fig. 5 that the electron bunches start to disperse and the main group of electrons drifts upward near saturation. In any bunch, the trajectories with positive slope indicate that the electrons are fast moving in an upward direction and tend to reach the accelerating phase. However, the 


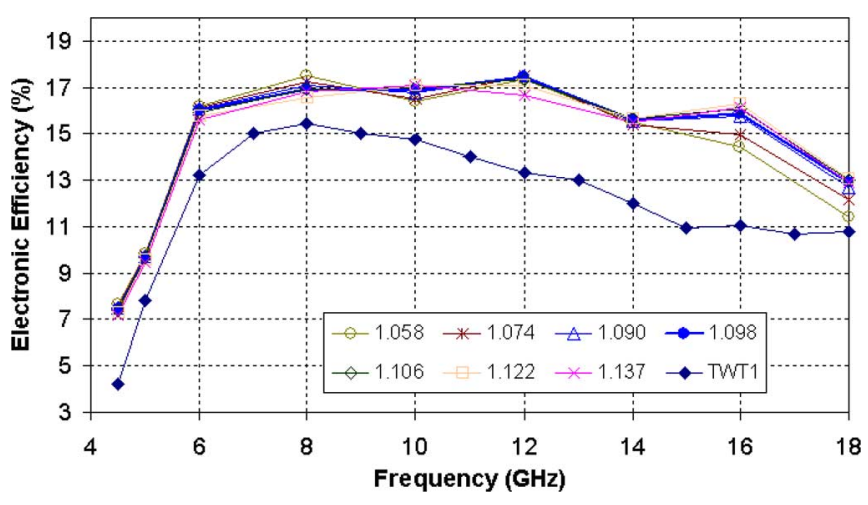

Fig. 6. Effect of final pitch variation on the electronic efficiency of the TWT with positive taper 2 .

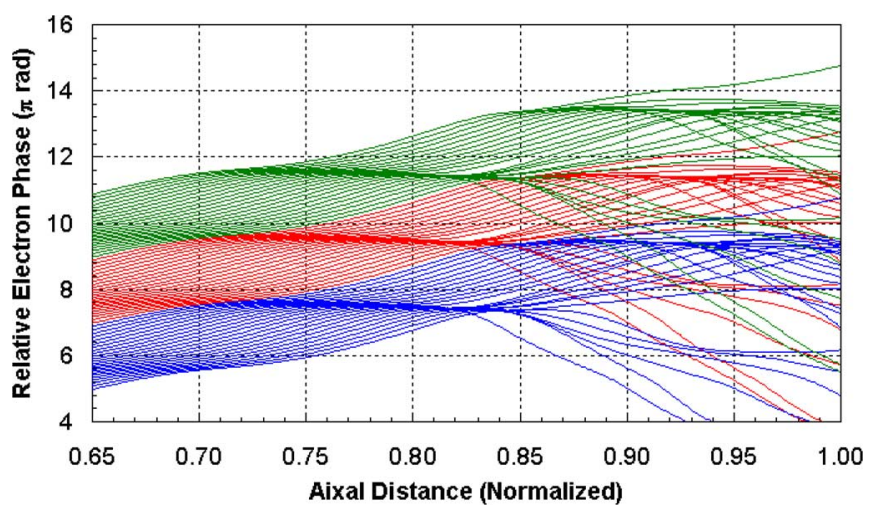

Fig. 7. Applegate diagram of the TWT with positive taper 2 and final taper pitch 1.098 (normalized) at $18 \mathrm{GHz}$.

electrons with negative slope reach the decelerating phase at saturation (normalized axial length 1.0) and transfer their energy to the RF wave. Clearly, the intention is for the majority of the trajectories to move downward so that they give up their energy to the wave. Based on the location of the bunch, two different starting positions along the axis were chosen as taper 1 and taper 2. Both tapers have the same initial and final pitch but different lengths. In both the cases, the initial pitch was kept the same as that of TWT1 and the final pitch was optimized through a number of iterations to achieve maximum efficiency. To show the effect of final pitch variation, the values of simulated electronic efficiency versus frequency are plotted in Fig. 6 for a number of different pitch values. For all computations, the taper length is the same as positive taper 2 , and pitch values are normalized with respect to the uniform pitch. None of the pitch values in the plot result in the highest electronic efficiency at both ends of the operating band. As a compromise, the bold line in the plot for normalized pitch value of 1.098 was chosen as optimized. The computed efficiency of this optimized positive pitch taper shows significant improvement over TWT1 throughout the band. To examine the nature of electron trajectories in the optimized configuration, an Applegate diagram at $18 \mathrm{GHz}$ is shown in Fig. 7. A comparison between Figs. 5 and 7 reveals that the bunch center is tending to move upward toward saturation in the former case, whereas in the latter case,

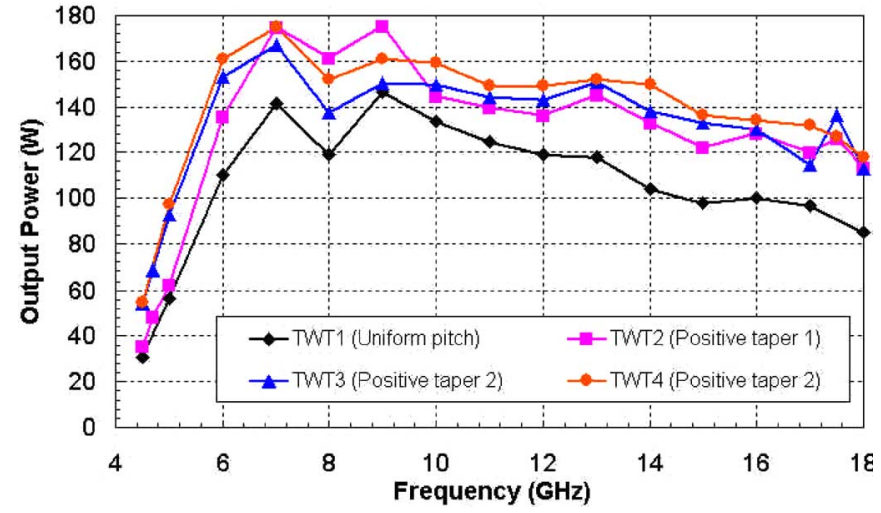

Fig. 8. Comparison of saturated fundamental output power.

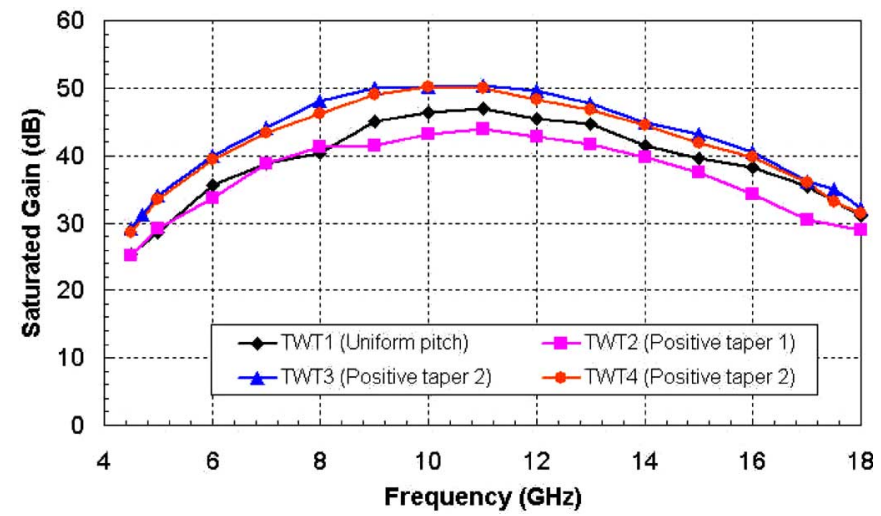

Fig. 9. Comparison of saturated gain.

it tends to move downward. The positive taper increases the phase velocity of the wave to resynchronize it with the velocity of those electrons, which are tending to be accelerated. This makes it possible to transfer more energy from the beam to the $\mathrm{RF}$ wave. Comparisons at other frequencies result in similar observations [20].

\section{EXPERIMENTAL EVALUATION}

Based on the positive taper designs, three different tubes, TWT2, TWT3, and TWT4, were developed in sequential order. After every iteration, the experimental results were analyzed and necessary design modifications were incorporated in the next iteration for further improvements in the performance. This strategy resulted in the need for fewer design iterations, facilitating lower cost and reduced time for development. Experimental results of saturated fundamental output power, saturated gain, and second harmonic content for all the four TWTs are plotted in Figs. 8, 9, and 10, respectively.

The salient points of these plots along with the configurations of the TWTs are discussed later.

\section{A. Comparison Between TWT1 and TWT2}

All components of TWT2 were the same as TWT1 other than the helix, which had a pitch profile similar to taper 1. A 


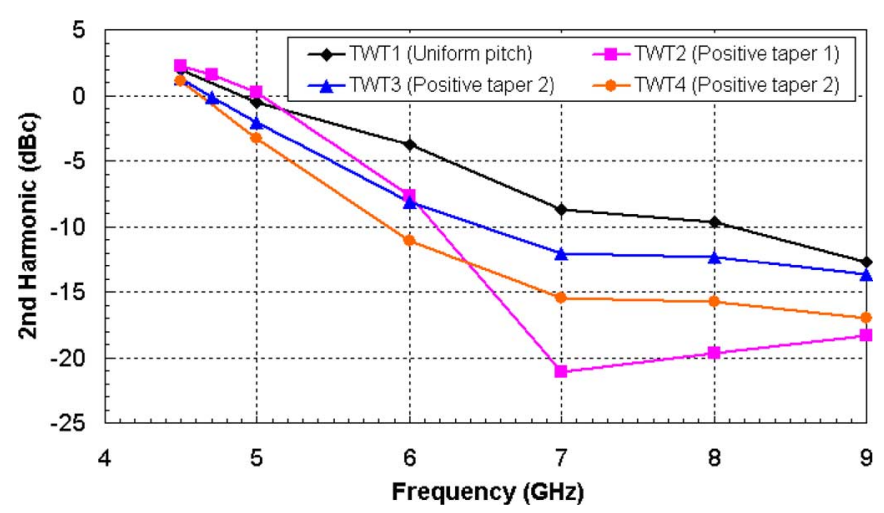

Fig. 10. Comparison of second harmonic power.

comparison between these two tubes shows that the saturated output power of TWT2 improves significantly in the frequency range $6-18 \mathrm{GHz}$ with very little or no change at the lower end of the operating band. The maximum and minimum improvements in this band are $42 \mathrm{~W}(1.3 \mathrm{~dB})$ at $8 \mathrm{GHz}$ and $11 \mathrm{~W}(0.35 \mathrm{~dB})$ at $10 \mathrm{GHz}$, respectively. However, the saturated gain of the tube decreases up to $5 \mathrm{~dB}$ in the frequency range $8-18 \mathrm{GHz}$ and remains unchanged at the lower end of the band. The second harmonic content of the device is lower at frequencies higher than $5 \mathrm{GHz}$, and it is lowest of all the four TWTs at frequencies higher than $6.5 \mathrm{GHz}$.

\section{B. Comparison Between TWT2 and TWT3}

To increase the output power over the entire operating band, especially at the lower end of the band, the helix pitch profile in TWT3 was changed to taper 2. To compensate for the loss of gain as observed in TWT2, the length of the attenuator was reduced. The new active length was calculated from the gain per unit length, where the gain includes the small-signal gain, and total loss. The rest of the components were kept the same as TWT2. A comparison between the two tubes shows a significant improvement in the output power throughout the band other than 7-10 GHz in TWT3. The saturated gain is also increased throughout the band. However, the second harmonic content becomes higher at the frequency range $6.5-9 \mathrm{GHz}$, though it is lower at the lower end of the operating band.

\section{Comparison Between TWT3 and TWT4}

TWT4 was developed with all components similar to TWT3 other than the electron gun, which was replaced with a new gun of larger cathode diameter. This reduces the cathode emission current density by nearly $45 \%$, and hence, increases the TWT operating life. The first three magnets of the periodic permanent magnet focusing structure were changed for profiling the magnetic field near the gun region to achieve optimized beam launching conditions. In comparison with TWT3, TWT4 shows slight improvement in the output power and second harmonic content over the entire band, while the saturated gain remains the same. The improvements are attributed to better beam laminarity, and hence, better beam-wave interaction. Maximum helix intercep-

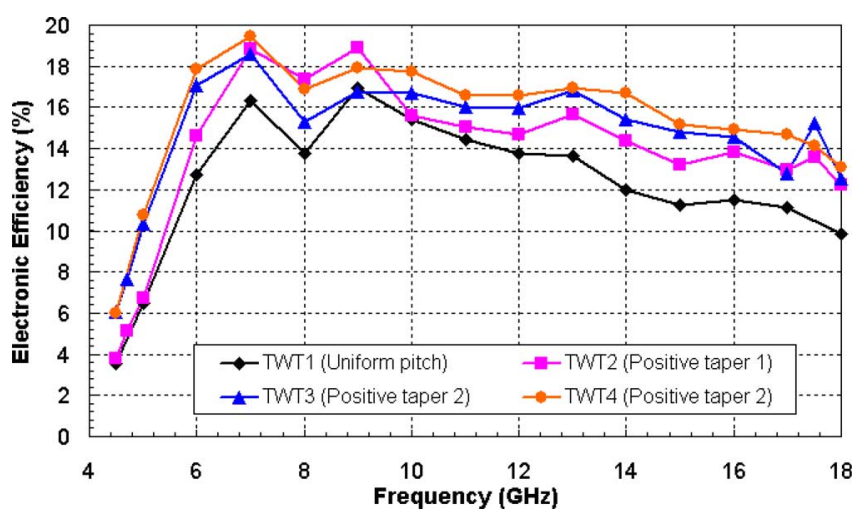

Fig. 11. Comparison of electronic efficiency.

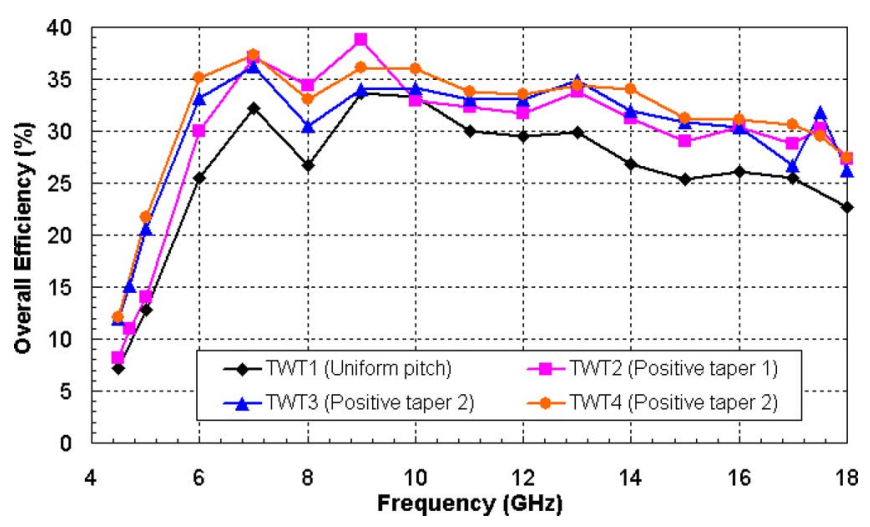

Fig. 12. Comparison of overall efficiency.

TABLE I

COMPARISON BETWEEN EXPERIMENTAL RESULTS OF TWT1 AND TWT4

\begin{tabular}{lcccc}
\hline Parameters & \multicolumn{4}{c}{ Improvements in TWT4 in comparison with TWT1 } \\
\cline { 2 - 5 } & Minimum & Frequency (GHz) & Maximum & Frequency (GHz) \\
\hline Output Power (W) & 14.5 & 9 & 50.3 & 6 \\
Saturated Gain (dB) & 0.3 & 18 & 5.8 & 8 \\
$2^{\text {nd }}$ Harmonic (-dBc) & 0.8 & 4.5 & 7.4 & 7 \\
Electronic Efficiency & 1.0 & 9 & 5.2 & 6 \\
(percentage points) & & & & \\
\hline
\end{tabular}

tion currents measured with RF are 13 and $10 \mathrm{~mA}$ for TWT3 and TWT4, respectively. In the case of dc operation, the beam transmission is better than $99 \%$ in both tubes. The consistency between the performance of TWT3 and TWT4 demonstrates the repeatability of the TWT manufacturing process.

The effectiveness of the positive phase velocity tapering in improving the electronic efficiency is demonstrated in Fig. 11. It is evident from the plot that all three positively tapered TWTs show significant improvement in performance over the TWT with uniform pitch. The overall efficiencies for all positively tapered TWTs were also improved by approximately 5\% points, as shown in Fig. 12. To summarize the experimental results, a comparison between the best performing tube, TWT4, and TWT1 is made in Table I, which is self-explanatory. Improvements up to $50 \mathrm{~W}(1.7 \mathrm{~dB})$ in output power and $5.2 \%$ points in electronic efficiency have been achieved. 


\section{CONCLUSION}

To improve broadband performance, a number of TWTs were designed and developed with helices of different taper lengths. The performance of three of these TWTs, which had shown significant improvements in output power and electronic efficiency over the entire operating band, was discussed. These three TWTs employed two different pitch taper lengths. Improvement in output power up to $1.7 \mathrm{~dB}$ and electronic efficiency up to $5.2 \%$ points were achieved. A close correlation between the experimental results of TWT3 and TWT4 proves that repeatability was maintained during the development. Future work will include the design and development of an optimized three-stage collector to improve the overall efficiency.

\section{ACKNOWLEDGMENT}

The authors would like to thank Dr. W. L. Menninger, L-3 Communications Electron Technologies, Inc., for his valuable suggestions to improve this paper. They also thank the Directors, e2v Technologies, U.K., for their support and for granting permission to publish this paper.

\section{REFERENCES}

[1] C. R. Smith, C. M. Armstrong, and J. Duthie, "The microwave power module: A versatile RF building block for high-power transmitters," Proc. IEEE, vol. 87, no. 5, pp. 717-737, May 1999.

[2] S. F. Paik, "Design formulas for helix dispersion shaping," IEEE Trans. Electron Devices, vol. ED-16, no. 12, pp. 1010-1014, Dec. 1969.

[3] J. L. Putz and M. J. Cascone, "Effective use of dispersion shaping in broadband helix TWT circuits," in IEDM Tech. Dig., Dec. 1979, pp. 422424.

[4] T. Onodera and W. Raub, "Phase velocity dispersion of a generalized metal-segment-loaded helix as used in broad-band traveling-wave tubes," IEEE Trans. Electron Devices, vol. 35, no. 4, pp. 533-538, Apr. 1988.

[5] B. N. Basu, Electromagnetic Theory and Applications in Beam-Wave Electronics. Singapore: World Scientific, 1996.

[6] C. L. Kory and J. A. Dayton, Jr., "Accurate cold-test model of helical TWT slow-wave circuits," IEEE Trans. Electron Devices, vol. 45, no. 4, pp. 966-971, Apr. 1998.

[7] A. S. Gilmour, Jr., M. R. Gillette, and J. T. Chen, "Theoretical and experimental TWT helix loss determination," IEEE Trans. Electron Devices, vol. ED-26, no. 10, pp. 1581-1588, Oct. 1979.

[8] P. K. Jain and B. N. Basu, "The effect of conductivity losses on propagation through the helical slow-wave structure of a traveling-wave tube," IEEE Trans. Electron Devices, vol. 35, no. 4, pp. 549-557, Apr 1988.

[9] S. Kapoor, R. S. Raju, R. K. Gupta, S. N. Joshi, and B. N. Basu, “Analysis of an inhomogeneously loaded helical slow-wave structure for broad-band TWT's," IEEE Trans. Electron Devices, vol. 36, no. 9, pp. 2000-2004, Sep. 1989.

[10] L. Kumar, R. S. Raju, S. N. Joshi, and B. N. Basu, "Modeling of a vane-loaded helical slow-wave structure for broad-band traveling-wave tubes," IEEE Trans. Electron Devices, vol. 36, no. 9, pp. 1991-1999, Sep. 1989.

[11] R. S. Raju, S. N. Joshi, and B. N. Basu, "Modeling of practical multioctave-band helical slow-wave structures of a traveling-wave tube for interaction impedance," IEEE Trans. Electron Devices, vol. 39, no. 4, pp. 996-1002, Apr. 1992.

[12] B. N. Basu, B. B. Pal, V. N. Singh, and N. C. Vaidya, "Optimum design of a potentially dispersion-free helical slow-wave circuit of a broad-band TWT," IEEE Trans. Electron Devices, vol. ED-32, no. 4, pp. 461-463, Apr. 1984.

[13] P. K. Jain and B. N. Basu, "The inhomogeneous dielectric loading effects of practical helix supports on the interaction impedance of the slow-wave structure of a TWT," IEEE Trans. Electron Devices, vol. 39, no. 3 , pp. 727-733, Mar. 1992.

[14] S. S. Jung, C. W. Baik, S. T. Han, S. G. Jeon, H. J. Ha, A. V. Soukhov, B. Jia, G. S. Park, H. S. Kim, H. S. Uhm, and B. N. Basu, "Wide-band semivane and heavily dielectric loaded helix traveling-wave tubes," IEEE Trans. Electron Devices, vol. 30, no. 3, pp. 1009-1016, Jun. 2002.

[15] C. Paoloni, "Analysis of dielectric rods with arbitrary shape for lowdispersion slow-wave structures in helix TWTs," IEEE Trans. Electron Devices, vol. 53, no. 6, pp. 1490-1493, Jun. 2006.

[16] V. Srivastava, R. G. Carter, B. Ravinder, A. K. Sinha, and S. N. Joshi, "Design of helix slow-wave structures for high efficiency TWTs," IEEE Trans. Electron Devices, vol. 47, no. 12, pp. 2438-2443, Dec. 2000.

[17] R. W. Gerchberg and K. B. Niclas, "The positively tapered traveling-wave tube," IEEE Trans. Electron Devices, vol. ED-16, no. 9, pp. 827-828, Sep. 1969.

[18] S. S. Jung, A. V. Soukhov, B. Jia, G. S. Park, and B. N. Basu, "Efficiency enhancement and harmonic reduction of wideband helix traveling-wave tubes with positive phase velocity tapering," Jpn. J. Appl. Phys., vol. 41, no. 6A, pp. 4007-4013, Jun. 2002.

[19] T. K. Ghosh, A. J. Challis, A Tokeley, K. Rushbrook, I. Poston, R. Matthews, A. Jacob, and D. Bowler, "Improvements in broadband performance of mini-TWTs at e2v technologies," in Proc. Int. Vac. Electron. Conf., Kitakyushu, Japan, 2007, pp. 31-32.

[20] T. K. Ghosh, Design of Positive Taper for 2 nd Generation Mini-TWTs. Chelmsford, U.K.: e2v Technologies, 2006.

[21] V. Srivastava and S. N. Joshi, "Improved nonlinear model for multisignal analysis of helix TWTs," in Proc. Inst. Electr. Eng. H, Apr. 1992, vol. 139, no. 2, pp. 129-134.

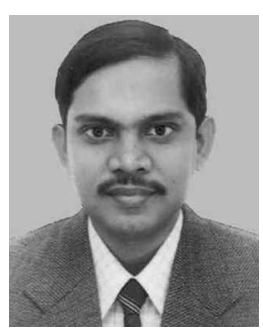

Tushar K. Ghosh (M'00) received the B.E. degree from Bengal Engineering and Science University, Shibpur, India, in 1990, and the M.E. degree from Jadavpur University, Kolkata, India, in 1993, both in electronics and telecommunication engineering, and the Ph.D. degree in engineering from the University of Lancaster, Lancaster, U.K., in 2002.

From 1993 to 1994 , he was a Senior Research Fellow at Jadavpur University, where he was engaged in research on electromagnetic pulse sensors. During 1995-2004, he was a Scientist at the Central Electronics Engineering Research Institute (CEERI), Pilani, India. He was a key member of the C-band and Ku-band space traveling-wave tube (TWT) project teams, where he was engaged in design and development activities. He has developed a number of computer codes for implementing advanced optimization techniques. Since 2004, he has been a Principal Engineer at e2v Technologies Ltd., Chelmsford, U.K., where he is currently engaged in improving the design and modeling techniques of TWTs. He is the author or coauthor of several papers published in journals and conference proceedings. His current research interests include vacuum device physics, modeling, and design.

Dr. Ghosh was the recipient of Commonwealth Scholarship from 1999 to 2002. He was one of the first three recipients of the IEEE-EDS Graduate Student Fellowship Award in 2001

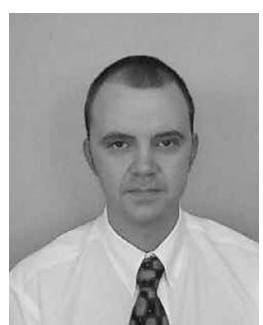

Anthony J. Challis received the H.N.C. degree in electromechanical engineering from Anglia Polytechnic University (APU), Chelmsford, U.K., in 1987.

Since 1983, he has been with e2v Technologies Ltd., Chelmsford, initially as an Apprentice in mechanical engineering, and later, as a Product Manager of traveling-wave tubes (TWTs) and microwave amplifier systems. He has been engaged in the development of new products and reengineering existing devices. He was at the Technical Authority for Helix TWTs. Achievements in electron gun and periodic permanent magnet (PPM) stack design led to his involvement in the successful development of a range of "mini TWTs" designed for airborne decoy applications. 


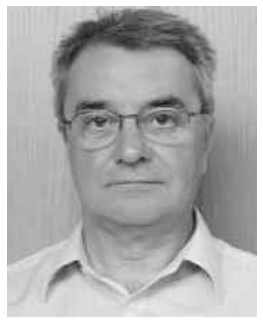

Alan Jacob received the Degree in physics from the City University, London, U.K., in 1976.

From 1976 to 1988, he was a Development Engineer in the Magnetron Section, Marconi-Osram (M-O) Valve Company, London, where he was engaged in producing a product line of miniature magnetrons. Since 1988, he has been with e2v Technologies, Chelmsford, U.K., initially as a Principal Engineer of the traveling-wave tube (TWT) Development Group, and later, as a Project Manager of helix TWT.

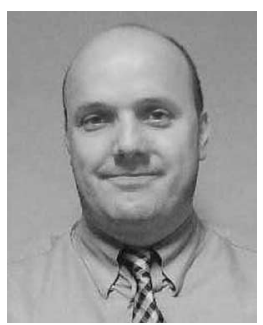

Darrin Bowler received the Degree in physics from the University of Warwick, Coventry, U.K., in 1989.

Since 1990, he has been with e2v Technologies Ltd., Chelmsford, U.K., where he was engaged in research on high-power klystrons for particle accelerator applications. Initially, he was the Engineering Group Leader in a number of microwave tubes and helix traveling-wave tubes (TWTs) projects that provide both production and design and development support. Currently, he is the Engineering Manager of Power Tubes and Systems.

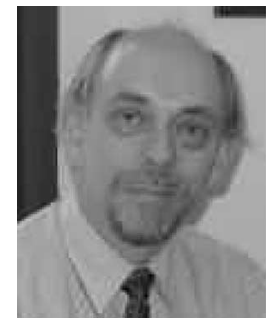

Richard G. Carter (M'97-SM'01) received the Degree in physics from the University of Cambridge, Cambridge, U.K., in 1965, and the Ph.D. degree in electronic engineering from the University of Wales, Cardiff, U.K., in 1968.

From 1968 to 1972, he was a Development Engineer at the English Electric Valve Company Ltd., Chelmsford, U.K., where he was engaged in research on high-power traveling-wave tubes (TWTs). During 1972, he was a Lecturer in the Department of Engineering, University of Lancaster, Lancaster, U.K., became a Senior Lecturer in 1986, and is currently a Professor of Electronic Engineering. His current research interests include electromagnetics and microwave engineering with particular reference to the theory, design, and computer modeling of microwave tubes and particle accelerators.

Prof. Carter is a Fellow of the Institution of Engineering and Technology (IET) and a member of the Vacuum Electronics and Compact Modeling Technical Committees of the IEEE Electron Devices Society. 